\title{
Role of Finite-Amplitude Rossby Waves and Nonconservative Processes in Downward Migration of Extratropical Flow Anomalies
}

\author{
Sandro W. Lubis, Clare S. Y. Huang, and Noboru Nakamura \\ Department of the Geophysical Sciences, University of Chicago, Chicago, Illinois \\ NOUR-EDDINE OMRANI \\ Geophysical Institute, University of Bergen, and Bjerknes Centre for Climate Research, Bergen, Norway \\ MARTIN JUCKER \\ School of Earth Sciences, and Australian Research Council Centre of Excellence for Climate System Science, \\ University of Melbourne, Melbourne, Victoria, Australia
}

(Manuscript received 4 December 2017, in final form 6 February 2018)

\begin{abstract}
There is growing evidence that stratospheric variability exerts a noticeable imprint on tropospheric weather and climate. Despite clear evidence of these impacts, the principal mechanism whereby stratospheric variability influences tropospheric circulation has remained elusive. Here, the authors introduce a novel approach, based on the theory of finite-amplitude wave activity, for quantifying the role of adiabatic and nonconservative effects on the mean flow that shape the downward coupling from the stratosphere to the troposphere during stratospheric vortex weakening (SVW) events. The advantage of using this theory is that eddy effects (at finite amplitude) on the mean flow can be more readily distinguished from nonconservative effects.

The results show (in confirmation of previous work) that the downward migration of extratropical wind anomalies is largely attributable to dynamical adjustments induced by fluctuating finite-amplitude wave forcing. The nonconservative effects, on the other hand, contribute to maintaining the downward signals in the recovery stage within the stratosphere, hinting at the importance of mixing and diabatic heating. The analysis further indicates that variations in stratospheric finite-amplitude wave forcing are too weak to account for the attendant changes and shapes in the tropospheric flow. It is suggested that the indirect effect of tropospheric finite-amplitude wave activity through the residual displacements is needed to amplify and prolong the tropospheric wind responses over several weeks. The results also reveal that the local tropospheric wave activity over the North Pacific and North Atlantic sectors plays a significant role in shaping the high-latitude tropospheric wind response to SVW events.
\end{abstract}

\section{Introduction}

There is clear evidence that stratospheric variability, for example, associated with a weakened polar vortex including major stratospheric sudden warmings (SSWs), can have substantial effects on surface weather and climate, particularly at middle to high latitudes in the winter (Baldwin and Dunkerton 1999, 2001; Baldwin

\footnotetext{
Supplemental information related to this paper is available at the Journals Online website: https://doi.org/10.1175/JAS-D-170376.s1.

Corresponding author: Sandro W. Lubis, slubis@uchicago.edu
}

et al. 2003; Mitchell et al. 2013; Kidston et al. 2015). The observed downward migration of extratropical wind anomalies in the Northern Hemisphere $(\mathrm{NH})$ was first shown by Kodera et al. (1990). In that process, the westerly wind anomalies first appear in the upper stratosphere and propagate downward into the troposphere, resulting in stronger tropospheric westerlies at high latitudes in the following weeks.

Much of the evidence for the downward dynamical coupling in the $\mathrm{NH}$ is manifested in terms of concurrent northern annular mode (NAM) anomalies between the stratosphere and troposphere (Baldwin and Dunkerton 1999, 2001; Baldwin et al. 2003). The NAM anomalies first appear in the stratosphere and subsequently progress 
downward into the troposphere over periods of a few weeks (Baldwin and Dunkerton 1999). With the comparatively longer memory of the lower stratosphere relative to the troposphere, signals of strong stratospheric variability can help to enhance the predictability of the tropospheric NAM on intraseasonal time scales (Baldwin et al. 2003; Scaife et al. 2014). Subsequent studies have shown that the best extended-range forecasts of the Arctic Oscillation (AO) in the extended winter period can be achieved by using the lower-stratospheric NAM index as a predictor (Baldwin et al. 2003; Tripathi et al. 2015).

A range of theories have been proposed to explain the observed stratosphere-troposphere dynamical coupling, including 1) downward migration induced by local, wavemean flow interaction (Plumb and Semeniuk 2003), 2) adjustment of the tropospheric flow to stratospheric potential vorticity (PV) anomalies (Hartley et al. 1998; Ambaum and Hoskins 2002; Black 2002), 3) a downward control principle (Haynes et al. 1991; Holton et al. 1995), 4) planetary wave reflection (Perlwitz and Harnik 2003; Shaw et al. 2010; Lubis et al. 2016, 2017, 2018), and 5) internal tropospheric eddy feedback (Wittman et al. 2004); however, the relative contributions of the various mechanisms are not yet fully understood, and inferences are still inconclusive (Kunz and Greatbatch 2013). In particular, it remains unclear how much the downward migration of wind anomalies from the stratosphere to the troposphere is attributable to forcing by wave transience of arbitrary amplitude (a change of local wave activity with time) and how much is due to nonconservative processes associated with eddy-induced mixing and diabatic forcing. Given the relatively long (intraseasonal) time scale involved, we anticipate that the latter will have significant influence in the evolution of the flow. In addition, it remains elusive how the effect of local wave activity at different altitudes contributes to the time evolution of downward-propagating anomalies and to the vertical structures of the responses.

To address these questions, we introduce the generalized finite-amplitude wave-mean flow interaction theory of Nakamura and Solomon (2010) as a novel diagnostic to quantify the role of finite-amplitude eddy and nonconservative processes in the observed stratospheretroposphere connection. The key difference between finiteamplitude wave activity theory and linear wave activity is that there are no higher-order terms that have to be neglected; hence, the nonconservative term can be readily quantified in its budget (Nakamura and Zhu 2010; Nakamura and Solomon 2010). Unlike several previous stratospheretroposphere coupling studies that solve the zonal-meanflow tendencies associated with a specified wave forcing (Song and Robinson 2004; Thompson et al. 2006; Kunz and Greatbatch 2013), our method follows the time evolution of the mean flow as the wave forcing is consistently adjusting to the changing mean state. Therefore, this allows us to quantify how much the mean flow is already modified by eddies at any given time. In addition, this diagnostic provides a precise link between wave activity and driving of the mean flow that remains true at finite wave amplitude so that the effect of wave forcing on the mean flow can be quantified more precisely even when the gradient of PV vanishes or material contours overturn (e.g., during SSW events).

The purpose of the current study is to quantify the net effects of finite-amplitude wave activity and nonconservative processes on the observed stratospheretroposphere connection during stratospheric vortex weakening (SVW) events, using gridded reanalysis data and the outputs of general circulation models (GCMs). The relative importance of finite-amplitude wave activity in the stratosphere and troposphere in controlling the time evolution of downward-propagation anomalies and the structure of the responses will also be examined in detail. In addition, the role of local finite-amplitude wave activity in the troposphere in shaping and maintaining the tropospheric wind response to SVW events is also investigated. In this study, the linear relationship between wave activity and the mean-flow adjustment is explored in the quasigeostrophic $(\mathrm{QG})$ framework throughout the paper. The QG dynamics permits easy interpretation, but the validity of the result in the tropics should be considered with caution, since the QG assumption breaks down in that region. Furthermore, we only consider wave activity that is limited to that of Rossby waves and exclude gravity wave contributions.

The paper is organized as follows. Section 2 provides the theory and data used for analyses. Section 3 describes the contribution of finite-amplitude eddies and nonconservative processes on the downward migration of the extratropical wind anomalies. In section 4, we assess the relative importance of wave activity in the stratosphere and troposphere separately. Finally, the role of local wave activity on the tropospheric wind response is given in section 5. The paper concludes with a summary and discussion in section 6 .

\section{Theory and data}

\section{a. Separation of adiabatic and nonconservative effects on the zonal-mean flow}

To quantify the effects of the advective process arising from the observed finite-amplitude eddies and the associated nonconservative processes on the mean flow, we employ a wave-mean flow interaction diagnostic based on finite-amplitude Rossby wave activity or negative angular pseudomomentum (Nakamura and Zhu 2010; Nakamura and Solomon 2010). The finite-amplitude wave activity $A^{*}$ is defined as the areal integrals of instantaneous azonality (area displacement) of the PV contours as 


$$
A^{*}\left(\phi_{e}, z, t\right)=\frac{1}{2 \pi a \cos \phi_{e}}\left[\iint_{q \geq Q\left(\phi_{e}\right)} q d S-\iint_{\phi \geq \phi_{e}} q d S\right],
$$

where $a$ is the radius of the planet, $q$ is QG PV, $Q\left(\phi_{e}\right)$ is $\mathrm{PV}$ with respect to equivalent latitude on each $z$ surface, and $d S=a^{2} \cos \phi d \lambda d \phi$ is the area element (see Fig. 1 for an illustration). The first surface integral in Eq. (1) covers the area north of the PV contour $q=Q\left(\phi_{e}\right)$ (red region in Fig. 1; the contour can be multiply connected), whereas the second integral covers the area north of latitude $\phi=\phi_{e}$ (green region). The equivalent latitude $\phi_{e}$ is defined such that the area covered by the two integrals is identical:

$$
\iint_{q \geq Q\left(\phi_{e}, z, t\right) ; 0.5 \pi \geq \phi} d S=\iint_{0.5 \pi \geq \phi \geq \phi_{e}} d S=2 \pi a^{2}\left(1-\sin \phi_{e}\right) .
$$

The QG PV is calculated as

$$
q=f+\zeta+\frac{f}{\rho_{0}} \frac{\partial}{\partial z}\left[\frac{\rho_{0}(\theta-\tilde{\theta})}{\partial \tilde{\theta} / \partial z}\right],
$$

where $f \equiv 2 \Omega \sin \phi$ is the Coriolis parameter, $\Omega$ is the rotation rate of the planet, $\zeta$ is relative vorticity, $z=-H \ln (p / 1000 \mathrm{hPa})$ is pressure pseudoheight, $\rho_{0} \propto e^{-z / H}$ is the background density, $H$ is the scale height, and $\tilde{\theta}$ is the layer mean potential temperature. The nonnegative quantity $A^{*}$ reduces to the linear pseudomomentum in the small-amplitude limit (Nakamura and Zhu 2010). The tendency of $A^{*}$ satisfies an exact Eliassen-Palm $(\mathrm{EP})$ relation as

$$
\frac{\partial A^{*}}{\partial t}+\nabla \cdot \mathbf{F}=S \simeq-\frac{K_{\mathrm{eff}}}{a} \frac{\partial Q}{\partial \phi_{e}}+\Delta \Sigma
$$

where $\nabla \cdot \mathbf{F}$ is the divergence of the EP flux and $S$ is the nonconservative sources/sinks of wave activity. The changes in $S$ can be accounted for by the changes in effective diffusivity of the irreversible mixing of PV across its own contour $K_{\text {eff }}$ (Nakamura 1996) and changes in the sources and sinks of wave activity as a result of diabatic heating and cooling averaged over the azonal (displaced) area of PV $(\Delta \Sigma)$. Fundamentally, when the terms on the right-hand side of Eq. (4) are zero, it takes the form of a conservation law for wave activity.

To obtain the exact relationship between finite-amplitude wave activity and zonal-mean zonal wind tendency, Eq. (4) is combined with the transformed Eulerian mean (TEM) zonal momentum equation (Andrews et al. 1987), and the following relationships are obtained [see Nakamura and Solomon (2010) for theoretical considerations]:
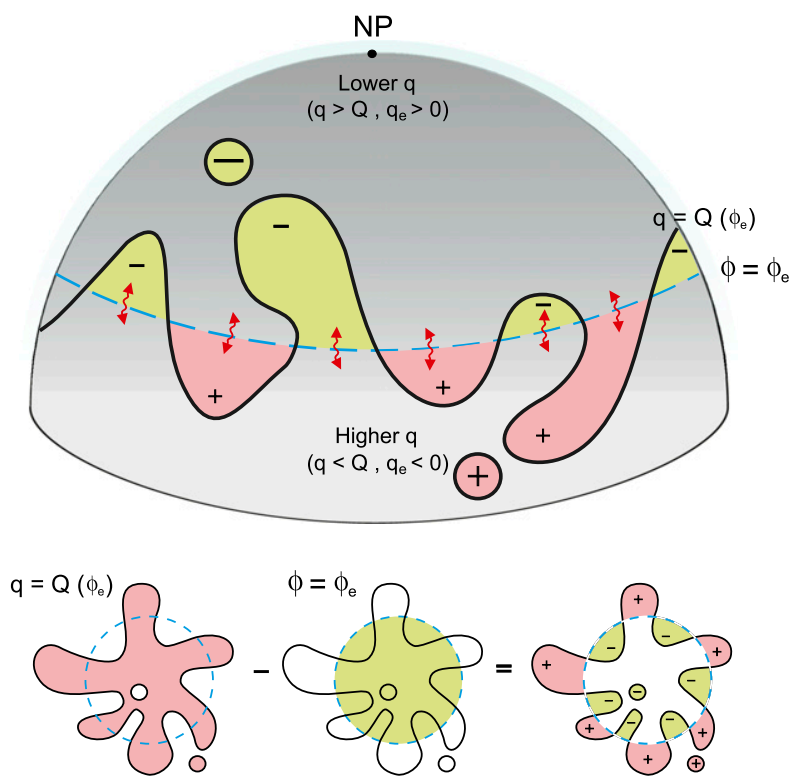

FIG. 1. Schematic presentation of the finite-amplitude wave activity on the sphere. Wave activity is defined as the difference in the areal integrals of instantaneous PV from zonal symmetry (over the red and below the green regions). The equivalent latitude $\phi_{e}$ is defined such that the red and the green areas are equal. The dashed contour is the latitude line $\phi=\phi_{e}$, and the solid contour is the line of $q=Q\left(\phi_{e}\right)$. The red arrows indicate the eddy fluxes producing the meridional areal displacement in the $Q$ contour.

$$
\begin{aligned}
& {\left[\frac{\partial^{2}}{\partial y^{2}}+\frac{1}{\rho_{0}} \frac{\partial}{\partial z}\left(\rho_{0} \varepsilon \frac{\partial}{\partial z}\right)\right]\left[\frac{\partial\left(\bar{u}-u_{\mathrm{REF}}\right)}{\partial t} \frac{\cos \phi_{e}}{f}\right]} \\
& \quad=-\frac{\partial^{2}}{\partial y^{2}}\left(\frac{\partial A^{*}}{\partial t} \frac{\cos \phi_{e}}{f}\right)
\end{aligned}
$$

and

$$
\begin{aligned}
& {\left[\frac{\partial^{2}}{\partial y^{2}}+\frac{1}{\rho_{0}} \frac{\partial}{\partial z}\left(\rho_{0} \varepsilon \frac{\partial}{\partial z}\right)\right]\left(\frac{\partial u_{\mathrm{REF}}}{\partial t} \frac{\cos \phi_{e}}{f}\right)} \\
& =-\frac{\partial^{2}}{\partial y^{2}}\left[(S+\bar{X}) \frac{\cos \phi_{e}}{f}\right]-\frac{\partial^{2}}{\partial y \partial z}\left(\frac{\overline{\mathfrak{J}}}{\partial \tilde{\theta} / \partial z} \cos \phi_{e}\right) .
\end{aligned}
$$

Here, $y=a \sin \phi_{e}, \varepsilon=f^{2} a^{2} H e^{\kappa z / H}\left(R \cos ^{2} \phi_{e} \partial \tilde{\theta} / \partial z\right)^{-1}, R$ is the gas constant, and $\kappa=R / c_{p}$, where $c_{p}$ is specific heat at constant pressure.

Equation (5) relates the wave activity density to the departure of the zonal-mean zonal flow $\bar{u}$ from a hypothetical eddy-free reference state flow $u_{\mathrm{REF}}$ (i.e., $\bar{u}-u_{\mathrm{REF}}=\Delta u$ ), while Eq. (6) shows that the reference state flow $u_{\mathrm{REF}}$ evolves only in response to nonconservative processes (including $S$, zonal-mean frictional forcing $\bar{X}$, and diabatic heating $\mathfrak{J}$ ), and hence, it filters out the effect of conservative (advective) eddy-mean flow interaction. Essentially, $u_{\mathrm{REF}}$ is the geostrophically balanced eddy-free flow corresponding to $\mathrm{QG} \mathrm{PV}$ in equivalent latitude $Q\left(\phi_{e}, z, t\right)$. 
Thus, $u_{\mathrm{REF}}$ can, in principle, be inverted from the PV gradient in equivalent latitude (Nakamura and Zhu 2010), although in this study we invert for $\Delta u$ [from Eq. (7)] and compute $u_{\mathrm{REF}}$ as $\bar{u}-\Delta u$.

A hypothetical adiabatic "adjustment" to the zonalmean wind relative to the reference state due to generation of the observed finite-amplitude eddies $\Delta u$ is obtained from the time integration of Eq. (5):

$$
\begin{gathered}
{\left[\frac{\partial^{2}}{\partial y^{2}}+\frac{1}{\rho_{0}} \frac{\partial}{\partial z}\left(\rho_{0} \varepsilon \frac{\partial}{\partial z}\right)\right]\left(\frac{\Delta u \cos \phi_{e}}{f}\right)} \\
=-\frac{\partial^{2}}{\partial y^{2}}\left(\frac{A^{*} \cos \phi_{e}}{f}\right) .
\end{gathered}
$$

Inverting Eq. (7) with proper boundary conditions gives us a net adiabatic effect of eddies on the mean flow $\Delta u$. Meanwhile, the contribution of the nonconservative terms on the mean flow can readily be diagnosed by $u_{\mathrm{REF}}=\bar{u}-\Delta u$.

The adjustment $\Delta u$ can be further partitioned into the direct effect of local wave activity $A^{*}$ and the indirect effect of the eddy-induced residual circulation $\Delta u_{R}$ :

$$
\Delta u=-A^{*}+\Delta u_{R} .
$$

Equation (8) states that the indirect effect of the eddyinduced residual circulation $\Delta u_{R}$ compensates the direct effect of local wave activity on the mean flow. By combining Eqs. (7) and (8), $\Delta u_{R}$ can be also inverted (using the same boundary condition as $\Delta u$ ) from the following relationship:

$$
\begin{gathered}
{\left[\frac{\partial^{2}}{\partial y^{2}}+\frac{1}{\rho_{0}} \frac{\partial}{\partial z}\left(\rho_{0} \varepsilon \frac{\partial}{\partial z}\right)\right]\left(\frac{\Delta u_{R} \cos \phi_{e}}{f}\right)} \\
=\frac{1}{\rho_{0}} \frac{\partial}{\partial z}\left[\rho_{0} \varepsilon \frac{\partial}{\partial z}\left(\frac{A^{*} \cos \phi_{e}}{f}\right)\right] .
\end{gathered}
$$

In this study, however, we directly computed $\Delta u_{R}$ as $\Delta u+A^{*}$ [Eq. (8)]. The projection of $A^{*}$ on $\Delta u$ and $\Delta u_{R}$ depends on the ratio of the horizontal scale of wave activity to the Rossby radius (Nakamura and Solomon 2010, 2011). For example, if the aspect ratio of wave activity is large, $\Delta u \approx-A^{*}$ so that the flow is decelerated by the wave activity and the residual circulation only plays a minor role (Nakamura and Solomon 2010).

\section{b. Piecewise inversion of finite-amplitude wave activity}

Since the relation in Eq. (7) is linear, the piecewise inversions by taking $A^{*}$ at different atmospheric levels can be performed unambiguously. Given observed $A^{*}$ at a certain reference level, we inverted Eq. (7) each day, where $A^{*}$ values at other levels are set to be zero or fixed toward daily climatological values (no significant differences using either assumptions because we are concerned about the departure of $\Delta u$ from the daily climatology). We restrict the domain to the $\mathrm{NH}$ and solve the discretized Eq. (7) numerically, with the following no-slip lower boundary condition for $u_{\mathrm{REF}}$ :

$$
\begin{aligned}
u_{\mathrm{REF}} & =0, & z & =0, \\
\Delta u & =\bar{u}, & z & =0, \\
\frac{\partial \Phi_{R}}{\partial z} & =\frac{\rho_{0}\left(\bar{u}+A^{*}\right) \cos \phi_{e}}{f}, & & =0,
\end{aligned}
$$

and the upper boundary condition following the thermal wind balance relation as

$$
\frac{\partial \Delta u}{\partial z}=-\frac{R}{f a H} \frac{\partial \Delta T}{\partial \phi_{e}}=-\frac{R \cos \phi_{e}}{f a H} \frac{\partial \Delta T}{\partial \sin \phi_{e}}
$$

or

$$
\frac{\partial}{\partial z}\left(\frac{\Delta u \cos \phi_{e}}{f}\right)=-\frac{R \cos ^{2} \phi_{e}}{f^{2} H} \frac{\partial \Delta T}{\partial y},
$$

where $\Delta T=\bar{T}-T_{\mathrm{REF}}$ and $T_{\mathrm{REF}}$ is the temperature field in thermal wind balance with $u_{\mathrm{REF}}$. We chose the no-slip lower boundary condition for $u_{\mathrm{REF}}$ because a nonzero surface zonal wind would imply the effect of angular momentum transport by the eddy against surface friction, a contradiction to the eddy-free assumption for the reference state. This boundary condition has been used successfully in numerous diagnostic studies of atmospheric phenomena (Nielsen-Gammon and Lefevre 1996; Hartley et al. 1998; Nakamura and Solomon 2010). An alternative lower boundary condition based on the adiabatic zonalization of surface temperature was also tested but was not employed for the current analysis (see the discussion and Fig. A1 in appendix A).

\section{c. Local finite-amplitude wave activity}

To understand the role of local $A^{*}$ in the observed downward migration of extratropical wind anomalies, we also implement a newly developed "local" finiteamplitude wave activity (LWA) diagnostic (Huang and Nakamura 2016). LWA is an extension of finiteamplitude wave activity as a function of longitude, which measures the net "exchange" of PV substance across latitude circles. In spherical coordinates, LWA is defined as

$$
\begin{aligned}
\tilde{A}^{*}\left(\lambda, \phi_{e}, z, t\right)= & -\frac{a}{\cos \phi_{e}} \int_{0}^{\Delta \phi_{e}} q_{e}\left(\lambda, \phi_{e}, \phi^{\prime}, z, t\right) \\
& \times \cos \left(\phi_{e}+\phi^{\prime}\right) d \phi^{\prime},
\end{aligned}
$$

where $\quad q_{e}\left(\lambda, \phi_{e}, \phi^{\prime}, z, t\right)=q\left(\lambda, \phi_{e}+\phi^{\prime}, z, t\right)-$ $q_{\mathrm{REF}}\left(\phi_{e}, z, t\right)$ is the eddy component of the QG PV $q$, 
defined as the departure from a zonally symmetric, Lagrangian-mean reference state $q_{\mathrm{REF}}$ [see Huang and Nakamura $(2016,2017)$ for theoretical details]. In the above, $\phi^{\prime}$ is the latitudinal displacement from $\phi$; $\Delta \phi_{e}(\lambda, \phi, z, t)$ is the meridional displacement of the contour $q=q_{\mathrm{REF}}$ from the latitude circle at $\phi$. LWA quantifies longitude-by-longitude contributions to finite-amplitude wave activity $A^{*}$ and thus describes eddy-mean flow interaction on a regional scale.

\section{d. Data}

ERA-Interim dataset (Dee et al. 2011) during 19792016 are used as observations of the wind, geopotential height, and air temperature with a horizontal resolution of $1.5^{\circ}$ latitude $\times 1.5^{\circ}$ longitude. In addition, to support the results of observationally based analysis, we also performed two free-running long-term GCM simulations with varying complexity (each 100 years): one with an idealized Geophysical Fluid Dynamics Laboratory (GFDL) dry dynamical core model (simple GCM) forced with realistic background temperature equilibrium $\left(T_{e}\right)$ fields obtained from the Rapid Radiative Transfer Model (RRTM) calculations (Jucker et al. 2013) and the other with NCAR's Community Earth System Model, version 1 (Whole Atmosphere Community Climate Model) [CESM1 (WACCM)], a stateof-the-art coupled chemistry-climate model (Gent et al. 2011; see supplemental section 1 for simulation details).

\section{Net contribution of finite-amplitude eddies and nonconservative processes}

Figures $2 \mathrm{a}-\mathrm{c}$ delineate the net contribution of eddies $\Delta u$ and nonadiabatic processes $u_{\mathrm{REF}}$ on zonal wind anomalies $\bar{u}$ during SVW events in the ERA-Interim (referred to simply as observations). The SVW event is defined similarly to Baldwin and Dunkerton (2001), by the dates on which the 50-hPa NAM values dropped below the threshold of -2 , while increasing in magnitude. We chose this particular vertical level as it is strongly coupled with tropospheric variability (Baldwin and Dunkerton 2001; Martineau and Son 2015). Our results are not sensitive to the exact reference level used to define the event.

By a cursory inspection of Fig. 2a, one can notice that there is a significant negative wind anomaly $\bar{u}$ that progresses downward from the upper stratosphere to the surface on a time scale of several weeks. This downward progression is reminiscent of a feature well known from composites of SSW events, although the time scales of such events are much shorter (about 1-2 weeks; Limpasuvan et al. 2004) than the polar vortex deceleration events, which take up to 5-7 weeks to progress from the upper into the lowermost stratosphere (Kunz and Greatbatch 2013). Since our events are defined based on the negative NAM index, the downward migration of the $\bar{u}$ anomaly in Fig. $2 \mathrm{~b}$ represents the signatures of both these events.

The results further indicate that the net adiabatic adjustments induced by finite-amplitude waves largely explain the downward migration of the $\bar{u}$ anomaly from the stratosphere to the troposphere (Fig. 2b), while the nonconservative effects primarily play a role in the aftermath or recovery within the stratosphere but seem negligible in the tropospheric evolution (Fig. 2c). In particular, in the upper stratosphere (between 1 and $10 \mathrm{hPa}$ ), the flow adjustment $\Delta u$ is everywhere negative from lag -10 to +10 days, indicating that the net effect of eddies on the mean flow is deceleration (Fig. 2b). It is consistent with the waves decelerating the flow in the upper stratosphere, and their subsequent propagation is capped at progressively lower altitudes, leading to the simultaneous descent of wave activity (see later in Fig. 3a) and $\bar{u}$ anomaly. After lag +10 days, there is a pocket of large positive $\Delta u$ associated with decreased wave activity. This counteracts the deceleration effect of nonconservative forcing on the mean flow $u_{\text {REF }}$ (Fig. 2c), indicating that a weakening of westerlies in that period is maintained by nonconservative processes.

In the middle to lower stratosphere (between 20 and $100 \mathrm{hPa}$ ), negative values of $\Delta u$ mostly explain the deceleration of $\bar{u}$ during and after the peak of the events (Figs. 2a,b). This is again consistent with increased wave breaking leading to a deceleration of the flow in the upper stratosphere, which results in the capping of wave activity successively at lower altitudes (see later in Fig. 3a). During the recovery period, the nonconservative processes maintain the negative $\bar{u}$ anomaly. This suggests that nonconservative processes, associated with the effects of dissipation on the waves and on the mean flow, play important roles in maintaining downward migration of the $\bar{u}$ anomaly during the recovery period. The nonconservative effects during the recovery of the SVW events can arise from the diabatic heating and increased eddy-induced mixing after the vortex breakdown, which altogether prevent a quick recovery of the polar vortex (Lubis et al. 2018, manuscript submitted to J. Atmos. Sci.; see also the discussion in section 6).

In the troposphere, the associated downward response is almost all adiabatic, as indicated by $-\bar{u} \simeq \Delta u$ (Fig. 2b). The contribution from nonconservative effects, on the other hand, seems to be negligible in tropospheric evolution (Fig. 2c), which contradicts previous work suggesting that the nonconservative process associated with frictional forcing (surface drag) acts to dampen the surface responses to downward stratospheric influence (e.g., Kunz and Greatbatch 2013). The reason for this is 

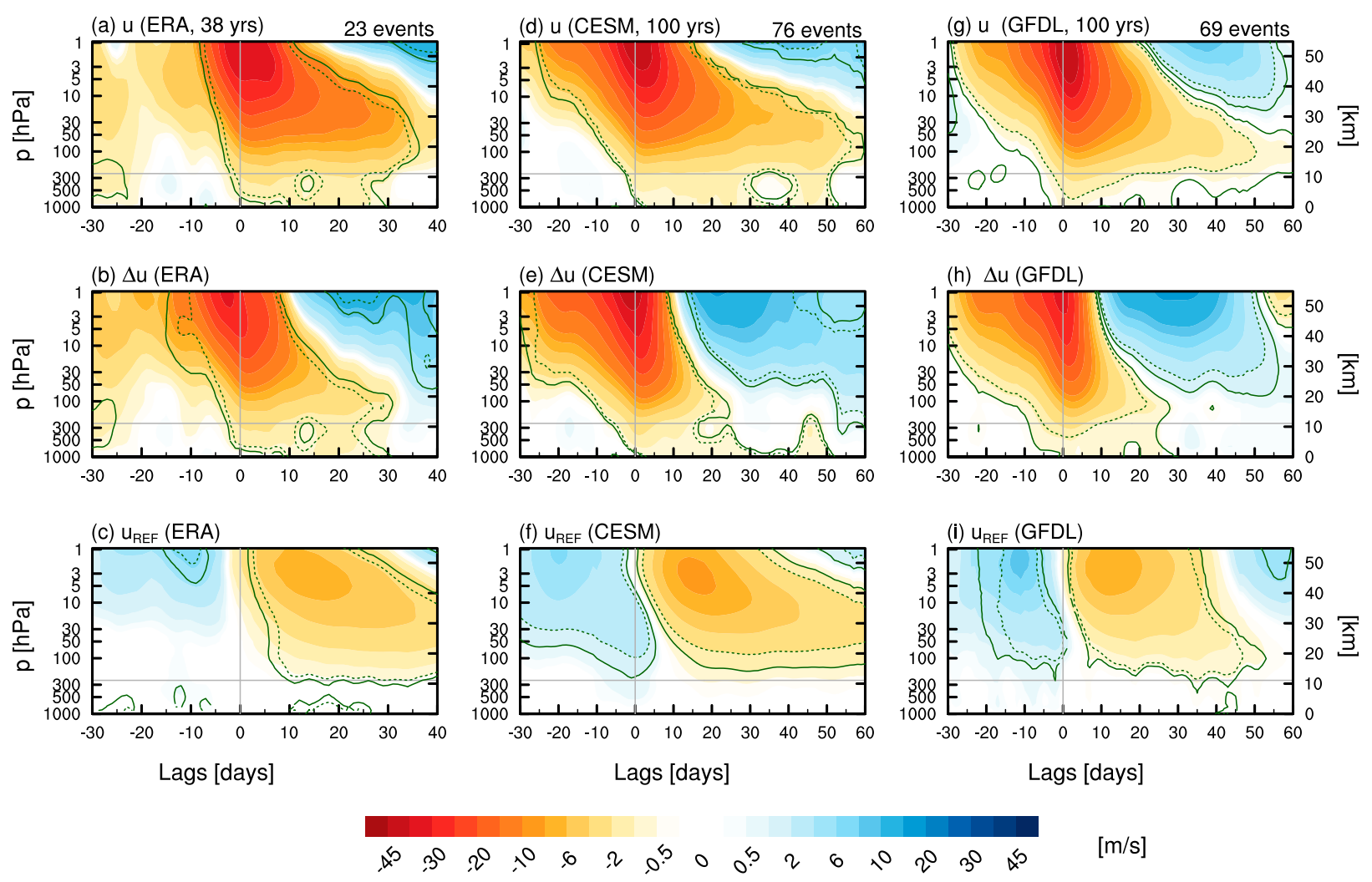

$[\mathrm{m} / \mathrm{s}]$

FIG. 2. The net contribution of adiabatic $(\Delta u)$ and nonconservative $\left(u_{\mathrm{REF}}\right)$ processes to the zonal-mean zonal wind anomalies $\bar{u}$ averaged over $50^{\circ}-80^{\circ} \mathrm{N}$ for the composites of SVW events in (a)-(c) 38-yr ERA-Interim (1979-2016) data, (d)-(f) 100-yr CESM1 (WACCM) fully coupled simulation, and (g)-(i) 100-yr GFDL dry dynamical core simulation forced by wave-2 topography and with realistic background temperature fields based on radiative transfer calculation. The thin horizontal lines indicate the approximate boundary between the troposphere and the stratosphere. Note that the red (blue) shading indicates negative (positive) wind anomalies. The solid (dashed) contour lines indicate the $95 \%$ (99\%) significance level based on a 1000-trial Monte Carlo test (see appendix B for more details).

the use of a no-slip surface boundary condition in our analysis, where $u_{\mathrm{REF}}$ is assumed to be steady or slowly evolving compared to the frictional time scale (i.e., $u_{\mathrm{REF}}=0$; Nakamura and Solomon 2010), obscuring the true magnitude of the surface drag effect on $u_{\mathrm{REF}}$. Nevertheless, the contribution of surface drag is relatively small compared to the tendency due to wave drag (cf., Kunz and Greatbatch 2013) so that the tropospheric wind response to SVWs is largely associated with adiabatic eddy-mean flow interactions.

The above results are found to be robust, in a qualitative sense, with respect to results from our GCM simulations (Figs. 2d-i), wherein we performed two long-term simulations (100 years each) with varying complexity: one with an idealized GFDL dry dynamical core model (simple GCM) and the other with NCAR's CESM1 (WACCM), a state-of-the-art, fully coupled chemistry-climate model. Although the time scale of the downward migration of zonal wind anomalies in the models is somewhat different from the observation (Figs. 2d,g), the general conclusion remains unchanged.
The adjustment $\Delta u$ largely explains the downward wind response in the stratosphere and the troposphere (Figs. 2e,h). On the other hand, the nonconservative processes dampen the effects of eddies before the peak of the events (lag 0$)$ in the stratosphere as indicated by a positive $u_{\text {REF }}$ anomaly, but it reinforces the downward wind response during the recovery period, particularly when wave activity becomes weaker (i.e., periods of decreased pseudomomentum density).

The results so far demonstrate that the downward migration of the wind anomalies is mainly due to the adiabatic adjustment induced by finite-amplitude wave activity. It is therefore interesting to further examine whether such adjustment $\Delta u$ is due to the direct (local) effect of wave activity or to the indirect effect of the eddy-induced residual displacements. Figure 3 illustrates the time-lagged composites of $-A^{*}$ and $\Delta u_{R}$ anomalies during SVW events from the observations. The results show that, in the stratosphere, about twothirds of the eddy forcing $-A^{*}$ is compensated by the Coriolis torque of the poleward residual displacement 

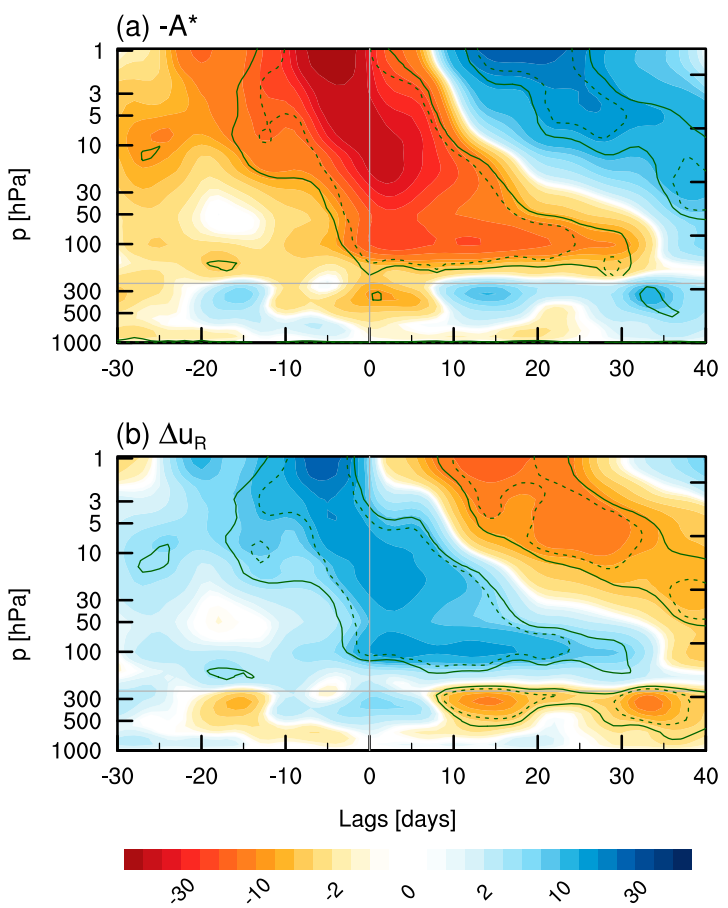

$[\mathrm{m} / \mathrm{s}]$

FIG. 3. Time-lagged composites of the observed (a) $-A^{*}$ and (b) $\Delta u_{R}$ anomalies averaged over $50^{\circ}-80^{\circ} \mathrm{N}$ from ERA-Interim. The solid (dashed) contour lines indicate the 95\% (99\%) significance level based on a 1000-trial Monte Carlo test. The thin horizontal lines indicate the approximate boundary between the troposphere and the stratosphere.

$\left(\Delta u_{R}=\Delta u-A^{*} ;\right.$ Fig. 3). Still, the downward migration of wind anomalies from the upper to the lower stratosphere is mainly driven by the direct effect of $A^{*}$ on the zonal-mean flow, consistent with the result of Plumb and Semeniuk (2003). Furthermore, during the onset of the tropospheric signal (from lag 0 to +10 days), the stratospheric $A^{*}$ anomalies show a near-instantaneous vertical coupling to the troposphere, although the statistical significance is lower than in the stratosphere. This result is consistent with previous work (e.g., Martineau and Son 2015), showing that stratospheric zonal wind anomalies exhibit a near-instantaneous vertical coupling to the troposphere during the onset of such events, which results from an anomalous upward and poleward propagation of planetary waves.

Somewhat surprisingly, after lag +10 days, the tropospheric $A^{*}$ anomaly turns negative, which is opposite from the stratosphere (Fig. 3a), and the residual torque also changes sign (Fig. 3b). In addition, $A^{*}$ does not descend directly from the stratosphere to the troposphere, although $\Delta u$ descends smoothly from the stratosphere to the troposphere (Fig. 2b). As we will show later (see Fig. 5), the tropospheric wind anomalies are largely attributable to wave activity anomalies in the troposphere, not those from the stratosphere. The decreased upper-tropospheric wave activity after 10 days is likely due to decreased baroclinic eddy activity after the jet migrates southward, assuming baroclinicity also shifts southward with the jet (e.g., Hitchcock and Simpson 2014; see also Fig. 4 below). This is consistent with the fact that wave activity in the upper troposphere is dominated by the signal of transient baroclinic eddies (Nakamura and Solomon 2010).

The negative wave activity anomalies in the upper troposphere are largely balanced by the negative residual torque anomalies, $A^{*} \approx \Delta u_{R}$, which is a solution of Eq. (9) in the limit of small (flat) aspect ratio. The weak negative wind anomalies $\Delta u$ arise from a slight surplus in $\Delta u_{R}$, which, as we will see below (refer to Fig. 5), are driven locally by the lower-tropospheric wave activity anomalies. These results clarify the previous stratosphere-troposphere coupling work based on idealized experiments, showing that the tropospheric responses are maintained locally by interactions with transient eddies (e.g., Song and Robinson 2004). Here, we further demonstrate that the process actually involves the indirect effect of the tropospheric eddyinduced residual displacements.

The vertical structure of $-A^{*}$ and $\Delta u_{R}$, as a function of latitude and height, is presented in Fig. 4. It can be seen that both magnitude and shape of the extratropical eddy-induced zonal wind anomalies $\Delta u$ from lag -10 to +5 days (during growing and maturation stages) are largely attributable to the direct (local) effect of wave activity (Figs. 4a-f). Such an effect also explains the northward-tilting structure in the high-latitude tropospheric wind anomaly $\left(45^{\circ}-60^{\circ} \mathrm{N}\right)$, which has not been examined in previous studies (see further discussion in section 5). On the other hand, during the recovery period in the stratosphere (and the maintenance stage in the troposphere; from lag +10 to +30 days), the deceleration of the tropospheric wind is mainly maintained by $\Delta u_{R}$ (Figs. $4 \mathrm{~g}-\mathrm{i}$ ), while $A^{*}$ opposes the effect of the eddy-induced residual displacements. These results highlight the important role of direct (local) and indirect effects of wave activity in setting the strength and shape of the zonal wind responses to SVW events.

\section{Relative roles of finite-amplitude wave activity in the stratosphere and troposphere}

The results so far indicate that the downward influence from the stratosphere to the troposphere during and after the onset of the SVW events is largely driven by the finiteamplitude Rossby wave activity (Fig. 2). The remaining analysis will focus on the mechanism that governs this propagation. We now consider the case of $\Delta u$, with the 

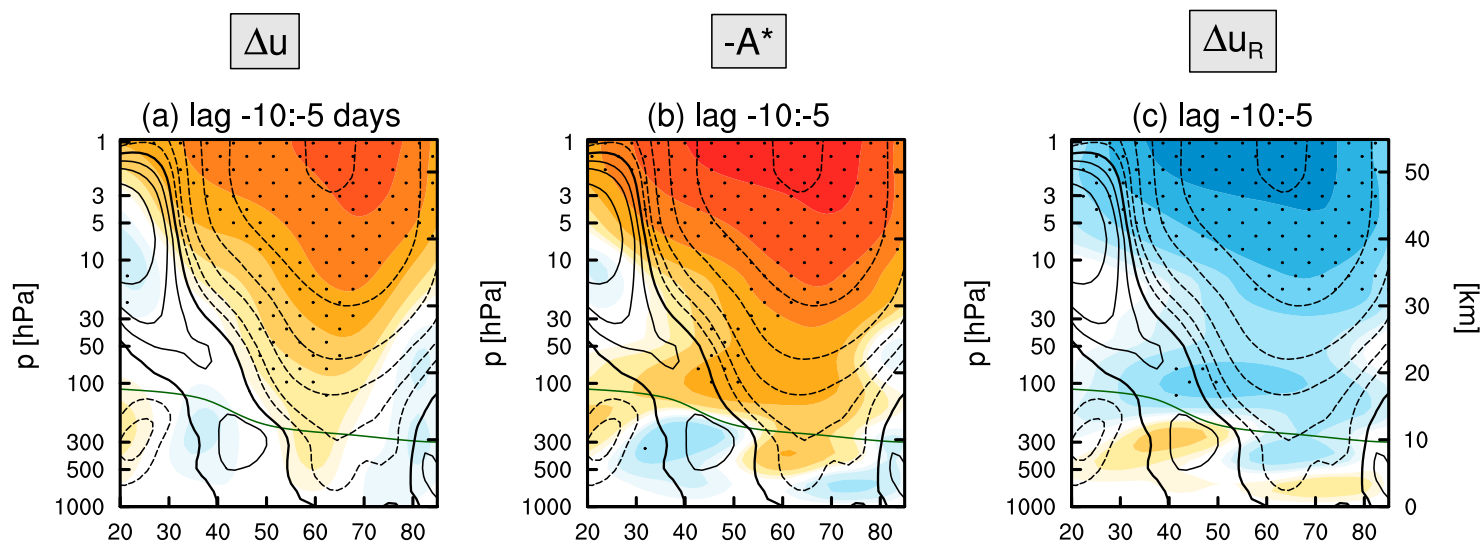

(d) lag 0:+5 days
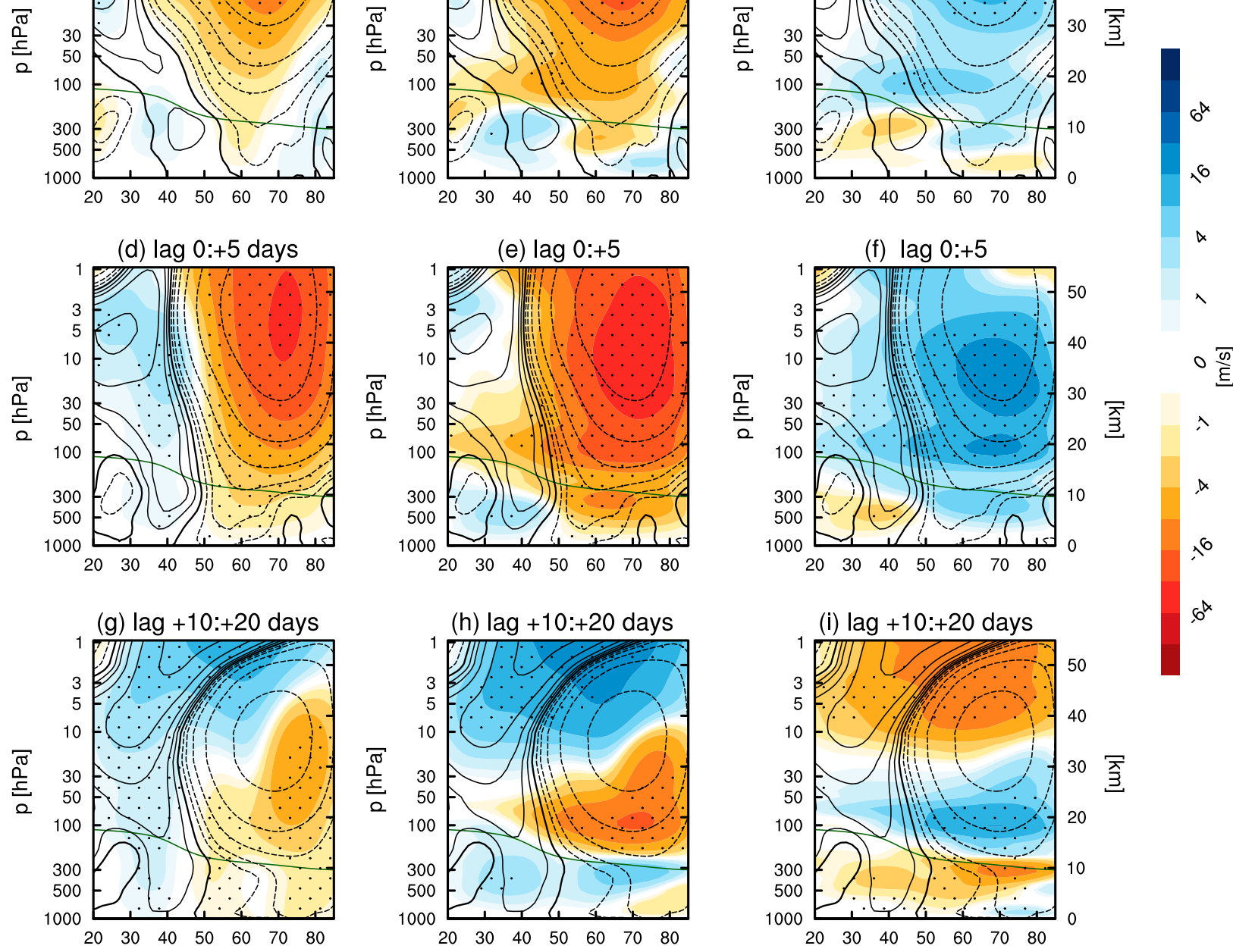

FIG. 4. Latitude-height composites of the (a),(d),(g) $\Delta u$,(b),(e),(h) $-A^{*}$, and (c),(f),(i) $\Delta u_{R}$ anomalies averaged between (top) lag -10 and -5 days, (middle) lag 0 and +5 days, and (bottom) lag +10 and +20 days during SVW events from ERA-Interim. Solid (dashed) lines indicate positive (negative) values of the zonal-mean wind anomalies. The contour intervals are in logarithmic powers of $2: \pm[0.5,1,2,4,8,16,32, \ldots] \mathrm{m} \mathrm{s}^{-1}$.

wave activity density $A^{*}$ restricted to either stratospheric or tropospheric parts. This allows us to quantify the relative significance of the finite-amplitude wave activity in the respective regions. In this analysis, we choose $250 \mathrm{hPa}$ as an approximate boundary between the troposphere and the stratosphere in the mid- to high latitudes, similar to Thompson et al. (2006).

\section{a. Intraseasonal eddy-induced propagation}

Figure 5 shows the relative importance of $A^{*}$ for the time scale of eddy-induced propagation during SVW events from the observations. We note that the zonal-mean wind anomaly shown in Fig. 5 includes only contributions from the finite-amplitude eddies. Inversions of Eq. (7) with $A^{*}$ field restricted to the upper stratosphere $(1 \leq p \leq$ $10 \mathrm{hPa}$ ) is displayed in Fig. 5a. It is evident that the upperstratospheric $A^{*}$ acts to initiate a downward-migrating signal from the upper to the middle stratosphere, from lag -10 to +10 days (Fig. 5a). The signals, however, do not propagate down to the troposphere.

The $A^{*}$ in the middle to lower stratosphere $(10<p \leq$ $250 \mathrm{hPa}$ ) explains the downward-migrating zonal wind anomalies from the stratosphere to the upper troposphere, with a time lag of 30 days (from -5 days at $20 \mathrm{hPa}$ 
Stratospheric $A^{*}$

(a) $\Delta \mathrm{u}$ for $\mathrm{A}^{*}[1 \leq \mathrm{p} \leq 10] \mathrm{hPa}$

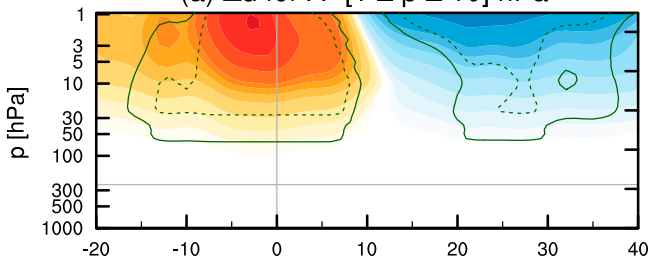

(b) $\Delta \mathrm{u}$ for $\mathrm{A}^{*}[10<\mathrm{p} \leq 250] \mathrm{hPa}$

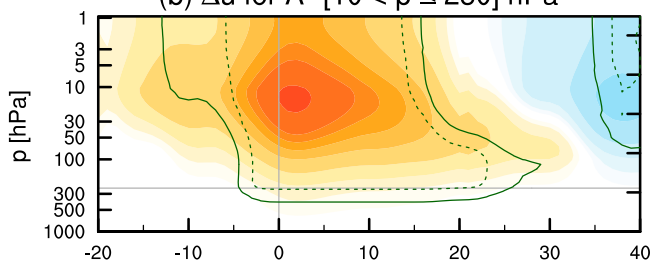

(c) $\Delta \mathrm{u}$ for $\mathrm{A}^{*}[1 \leq \mathrm{p} \leq 250] \mathrm{hPa}$

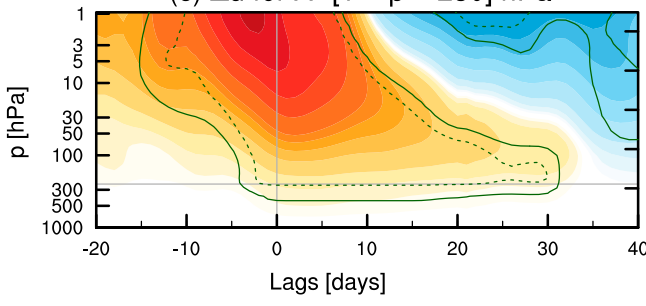

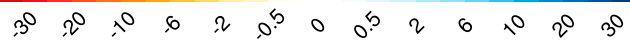

Tropospheric $A^{*}$

(d) $\Delta \mathrm{u}$ for $\mathrm{A}^{*}[250<\mathrm{p} \leq 500] \mathrm{hPa}$

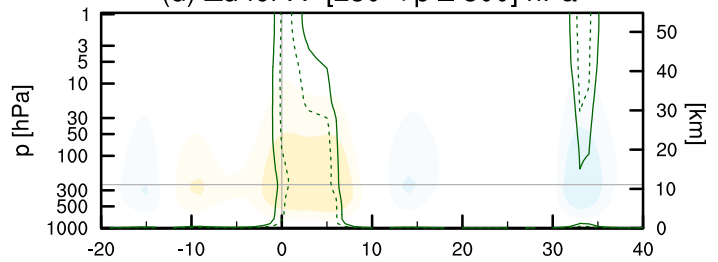

(e) $\Delta u$ for $A^{*}[500<p \leq 1000] \mathrm{hPa}$

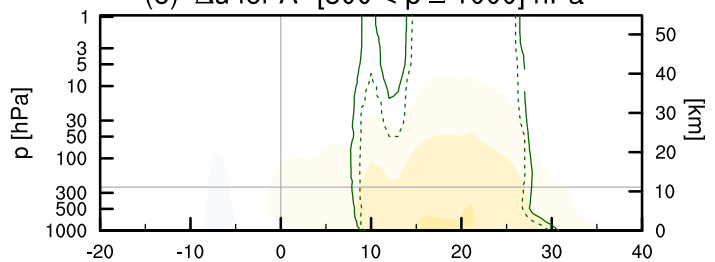

(f) $\Delta u$ for $A^{*}[250 \leq p \leq 1000] \mathrm{hPa}$

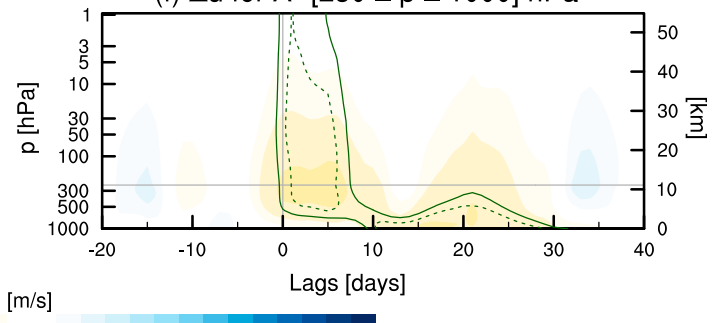

$[\mathrm{m} / \mathrm{s}]$

FIG. 5. Time-lagged composites of the observed zonal-mean zonal wind anomalies induced by eddies $\Delta u$ (as in Fig. 2b) resulting from the inversions of $A^{*}$ (a)-(c) at three different stratospheric layers (upper, middle, and entire stratosphere, respectively) and (d)-(f) at three different tropospheric layers from ERA-Interim. The solid (dashed) contour lines indicate the $95 \%$ (99\%) significance level based on a 1000-trial Monte Carlo test. The thin horizontal lines indicate the approximate boundary between the troposphere and the stratosphere.

to $300 \mathrm{hPa}$ at lag +25 days; Fig. $5 \mathrm{~b})$. It is also evident that midstratospheric $A^{*}$ induces upward influences, suggesting that negative $\Delta u$ in the upper stratosphere is partially driven by the lower-stratospheric wave activity.

Overall, the stratospheric $A^{*}$ plays a significant role in initiating and shaping downward-migrating signals in the stratosphere (from lag -10 to +25 days; Fig. $5 \mathrm{c}$ ). This dynamical adjustment $\Delta u$ is largely confined to stratospheric altitudes, and the most persistent signals are located in the lower stratosphere, with a duration of 31 days (from lag -5 to +25 days). These results support the previous work of Plumb and Semeniuk (2003) based on an idealized experiment, showing that the downward migration is largely dependent on local wave-mean flow interaction. In addition to that process, we also show that by imposing the wave forcing in the middle atmosphere only, the adjustment $\Delta u$ can propagate farther down to the upper troposphere (although the magnitude is relatively weak) and up to the upper stratosphere (Fig. 5b). This suggests an indirect influence of the wave activity to the upper-tropospheric and upperstratospheric flow. Nevertheless, it is evident that the stratospheric $A^{*}$ alone is too weak to account for the attendant changes in the tropospheric flow.

We further analyze the role of tropospheric $A^{*}$ for the downward propagation of $\Delta u$ (Figs. $5 \mathrm{~d}-\mathrm{f}$ ). The results indicate that $A^{*}$ in the upper troposphere (between 250 and $500 \mathrm{hPa}$ ) plays a significant role in maintaining lower-tropospheric and surface signals from lag 0 to +9 days (Fig. 5 d). On the other hand, solving the inversion for the wave activity in the layer $500<p \leq$ $1000 \mathrm{hPa}$ illustrates the role of lower-tropospheric eddy feedback in the maintenance of the surface response at lags $\sim 10-30$ days (Fig. 5e), which is more clearly shown than in the previous work. We also note that both upperand lower-tropospheric $A^{*}$ also affect the stratospheric wind responses. Overall, the results indicate that tropospheric $A^{*}$ (Fig. 5f) plays a significant role in amplifying and maintaining the tropospheric wind responses to SVW events. 
(a) Total u

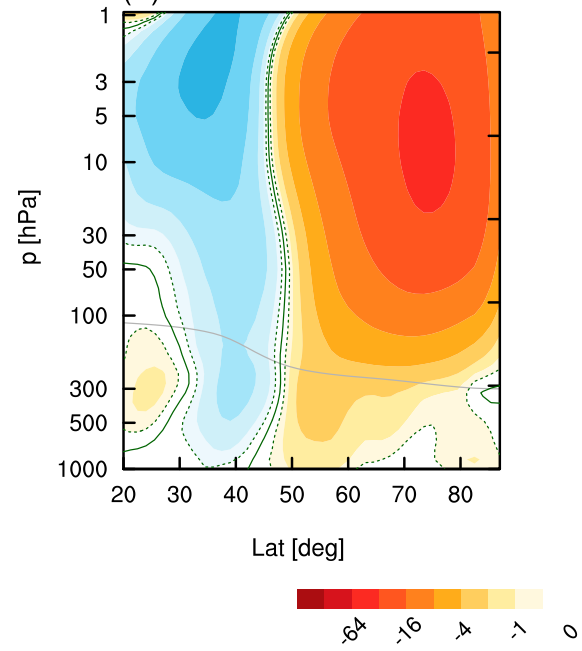

(b) $\Delta \mathrm{u}$ for total $\mathrm{A}^{*}$

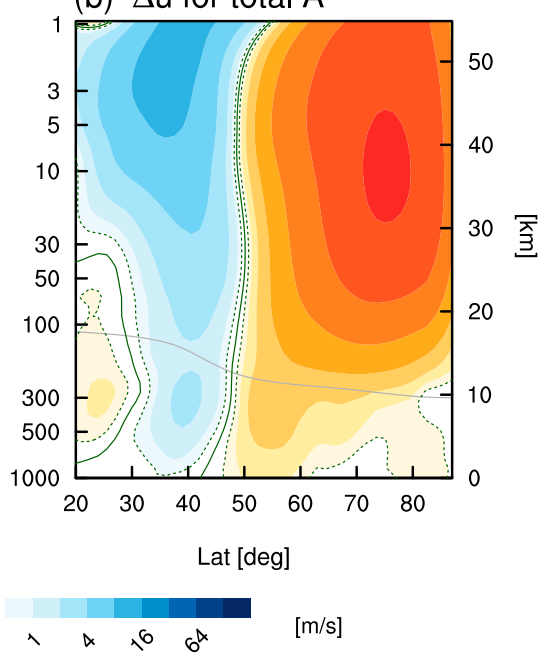

FIG. 6. Latitude-height composites of the (a) zonal-mean zonal wind anomaly and (b) eddy-induced zonal wind anomaly (obtained from the inversion of $A^{*}$ over the entire atmospheric column), averaged between lag 0 and +5 days during SVW events from ERAInterim. The gray solid lines indicate the approximate boundary between the troposphere and the stratosphere.

Considering both stratospheric and tropospheric adjustments $\Delta u$ together (Fig. 5), we see that 1) the tropospheric wind response is not the consequence of the direct stratospheric wave forcing and 2) the tropospheric adjustment $\Delta u$ is mainly attributed to the indirect effect of tropospheric eddy adjustment on zonal-mean flow through residual displacement after 10 days (Figs. 3 and 4). The stratospheric $A^{*}$ alone is too weak to account for the attendant changes in the tropospheric flow (Figs. 5a-c). The results support the previous modeling study by Kunz and Greatbatch (2013), that tropospheric wave forcing is important to maintain and prolong the tropospheric signals over several weeks (Figs. 5d-f). However, we emphasize here that it is the indirect effect of tropospheric wave activity through residual displacement that maintains this tropospheric wind response.

\section{b. Latitude-height structure of zonal-mean response}

We next analyze the observed two-dimensional structure of the wind response during the period when the stratosphere is strongly coupled with the troposphere (i.e., lag 0 to lag +5 days; Fig. 6). It is evident from Figs. $6 \mathrm{a}$ and $6 \mathrm{~b}$ that a large part of the zonal wind anomaly pattern, and its magnitude, are attributable to adiabatic eddy adjustments, which include "quasi barotropic" responses from the upper to lower stratosphere and a substantial northward-tilting structure in the highlatitude troposphere. The small residual of the difference between Figs. $6 \mathrm{a}$ and $6 \mathrm{~b}$ indicates the contribution of nonconservative effects on the mean flow during that period. This overall pattern is consistent with a wellknown structure of the zonal wind perturbations associated with the AO during $\mathrm{NH}$ winter (Baldwin and Dunkerton 1999; Black 2002).

Subsequently, we partition the $A^{*}$ field into stratospheric and tropospheric parts and again apply piecewise inversions separately to deduce the associated zonal wind field (Fig. 7). We note that since the relationship between wave activity and the mean-flow adjustment $\Delta u$ is linear, the sum of the fields plotted in Figs. $7 \mathrm{a}$ and $7 \mathrm{~b}$ is identical with Fig. 7c. It is evident that a large part of the stratospheric zonal wind anomaly is attributable to stratospheric $A^{*}$ (Figs. 7a-c); however, only $A^{*}$ in the middle to lower stratosphere contributes significantly to the observed high-latitude uppertropospheric wind anomalies (Fig. 7b). This implies that $A^{*}$ in the upper stratosphere is not necessarily required to diagnose a direct downward influence of stratospheric $A^{*}$ upon the troposphere (Fig. 7a); however, we note that a downward influence to the troposphere induced by stratospheric $A^{*}$ neither reaches the surface nor possesses the vertical tilt observed in Fig. 6. This suggests that a dynamical adjustment induced by eddies in the troposphere is required to shape the stratospheric downward influence into the troposphere.

As evidence for that, our results further show that tropospheric $A^{*}$ contributes significantly to shaping and amplifying the tropospheric wind response, which include the northward-tilting structure in the high-latitude wind anomaly between $45^{\circ}$ and $60^{\circ} \mathrm{N}$ and the tropospheric part 

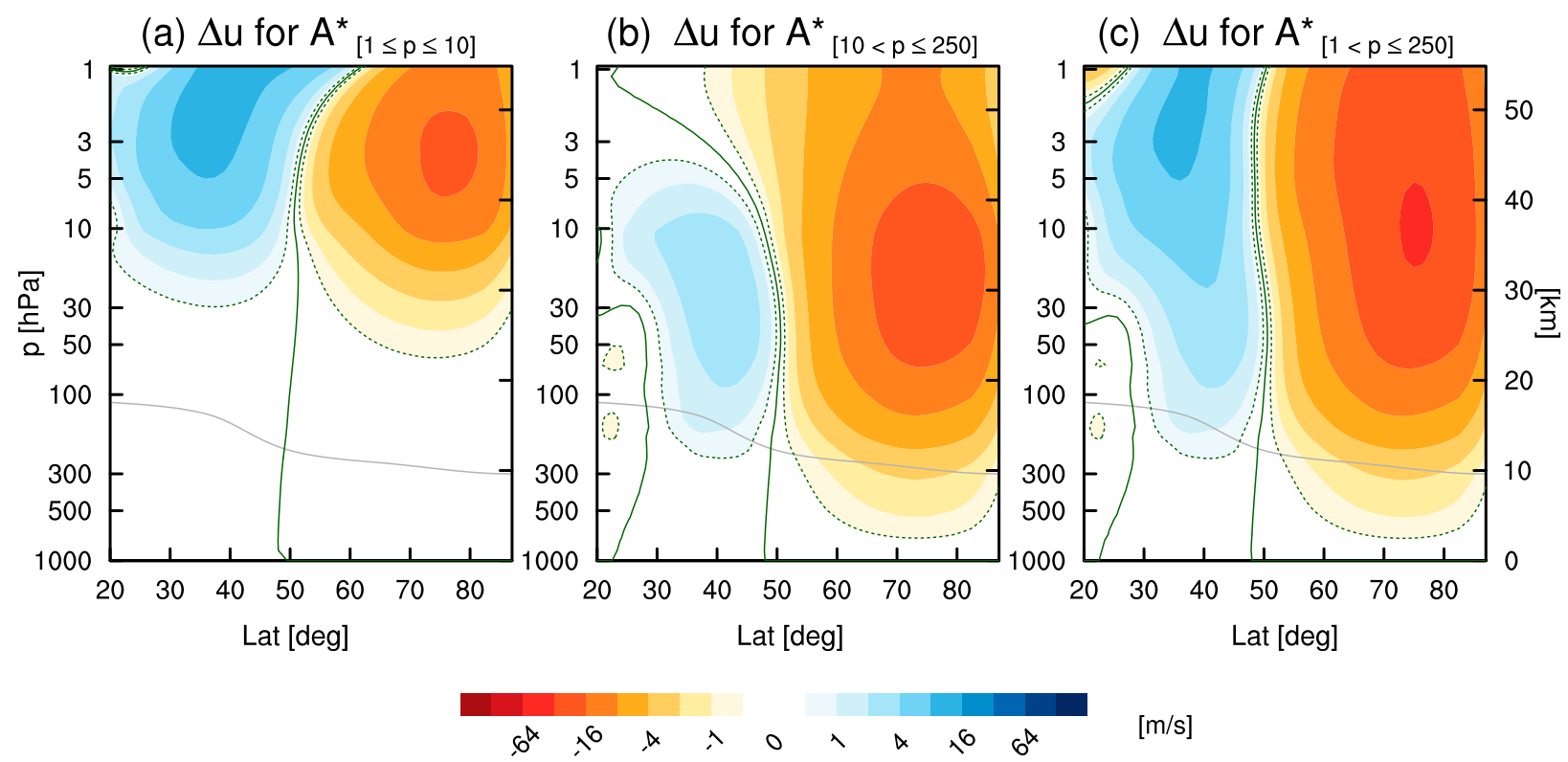

$[\mathrm{m} / \mathrm{s}]$

FIG. 7. Latitude-height composites of the observed zonal-mean zonal wind anomalies induced by eddies $\Delta u$ averaged between lag 0 and +5 days, resulting from the inversions of $A^{*}$ (a) in the upper stratosphere (1-10 hPa), (b) in the lower stratosphere (10-250 hPa), and (c) over the entire stratosphere $(1-250 \mathrm{hPa})$ from ERA-Interim. The gray solid lines indicate the approximate boundary between the troposphere and the stratosphere.

of the low-latitude wind anomaly between $20^{\circ}$ and $40^{\circ} \mathrm{N}$ (Figs. 8a-c). Such adjustment is largely maintained by the lower-tropospheric $A^{*}$ (Fig. 8b). The importance of the lower-tropospheric $A^{*}$ in shaping the northward-tilting structure of tropospheric wind response to the SVW event has been overlooked in previous studies.

\section{Relative role of finite-amplitude local wave activity}

It is interesting to further investigate what drives and maintains the observed northward-tilting structure in the high-latitude tropospheric wind anomaly when the stratosphere is strongly coupled to the troposphere (Figs. 6 and 8c). Previous studies have shown that such a pattern is associated with the $\mathrm{AO}$ in the troposphere during the NH winter (Baldwin and Dunkerton 1999; Black 2002); however, the mechanism behind this phenomenon has remained unclear.

We attempt to understand this by applying a newly developed LWA diagnostic of Huang and Nakamura (2016). Figures 9a and 9b show the composite of LWA before and after the peak of the SVW events in the observations. The analysis reveals that during the onset of these events, there is a strengthening of LWA in the North Pacific and North Atlantic, confined to the northern flank of the storm-track regions. The enhanced LWA leads to a weakening of the zonal wind in high latitudes (i.e., as expected from the theory where the change in zonal-mean zonal wind is anticorrelated with the change in wave activity amplitude). This feature is consistent with an equatorward shift of the tropospheric jet after the onset of the SVW events, which bears a striking resemblance to the surface signature of negative NAM (Limpasuvan et al. 2004; Baldwin and Dunkerton 1999).

Since the zonal average of LWA recovers finiteamplitude wave activity (Nakamura and Solomon 2010; Huang and Nakamura 2016), we can thus directly quantify the tropospheric zonal wind responses associated with tropospheric LWA in different regions. We chose three regions with the most distinct LWA activities during events, including the North Pacific, North Atlantic, and Eurasian sectors (Figs. 9c-e). We then applied the inversion by using a particular tropospheric $A^{*}$ (calculated from the zonally averaged LWA in each region) to deduce the associated zonal wind responses.

The results of this calculation are shown in Figs. 9c-e, which shed light on how the downward stratospheric influence is communicated to the tropospheric wind via LWA. It is evident that the northward-tilting structures in the high-latitude wind anomalies are attributable to tropospheric LWA forcing over the North Pacific and North Atlantic storm-track regions with comparable magnitudes (Figs. 9c and 9d). In contrast, the dynamical adjustments induced by the Eurasian LWA are not capable of reproducing the observed tropospheric wind structures correctly, characterized by quasi-barotropic wind responses in the troposphere, confined to the high 
(a) $\Delta u$ for $A^{*}$

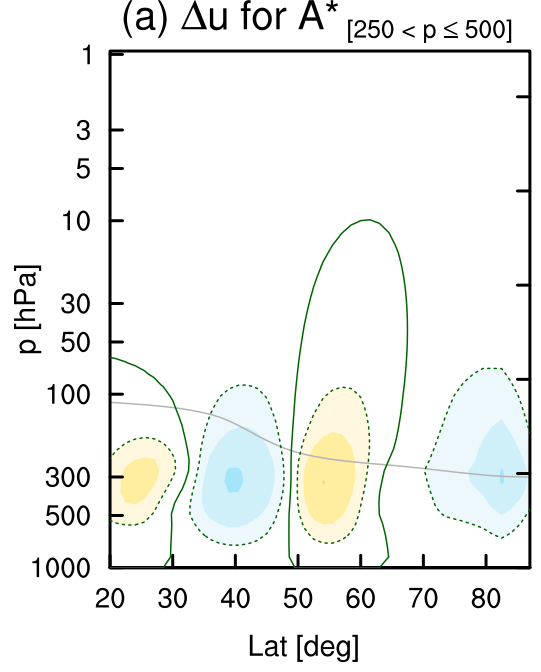

(b) $\Delta \mathrm{u}$ for $\mathrm{A}^{*}$

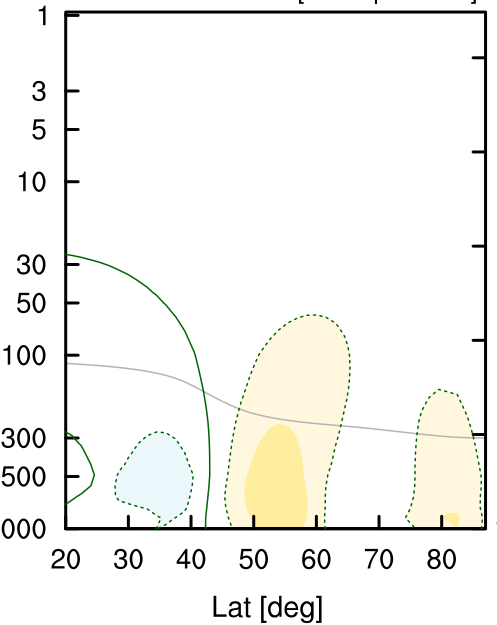

(c) $\Delta \mathrm{u}$ for $\mathrm{A}^{*}$

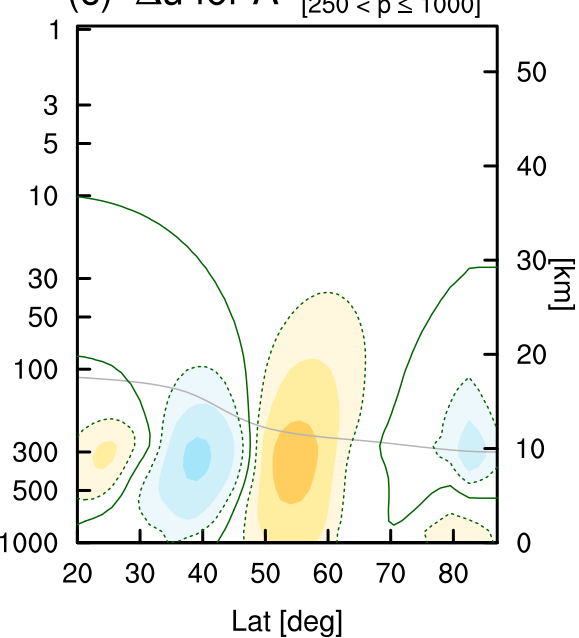

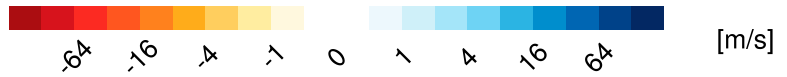

FIG. 8. As in Fig. 7, but for the inversions of $A^{*}$ (a) in the upper stratosphere (250-500 hPa), (b) in the lower troposphere (500-1000 hPa), and $(\mathrm{c})$ over the entire troposphere $(250-1000 \mathrm{hPa})$ from ERA-Interim. The gray solid lines indicate the approximate boundary between the troposphere and the stratosphere.

latitudes (poleward of $70^{\circ} \mathrm{N}$; Fig. 9e). This is consistent with the fact that there is not much enhancement in the tropospheric LWA over Eurasia after the peak of the SVW events (it is actually decreasing), so it does not lead to a deceleration of jet. Enhanced LWA in the storm-track regions is indicative of the important role of internal tropospheric baroclinic eddy feedbacks in driving the tropospheric wind changes. Further study will be necessary to ascertain the dynamical linkage that exists between LWA drivers and the local dynamical adjustments on the tropospheric zonal flow, for example, by implementing an LWA budget diagnostic of Huang and Nakamura (2017), to understand the relative importance of low-level meridional heat flux, horizontal convergence of wave activity flux, and nonconservative forcing in driving LWA.

The contribution of the dynamically active LWA in the Pacific and Atlantic regions in shaping tropospheric wind response to downward stratospheric signals has not been examined in previous studies and thus is worth further investigation. These results imply that proper simulation of AO behavior requires an atmospheric model that, at the very least, adequately represents LWA around the North Pacific and North Atlantic storm-track regions.

\section{Conclusions and discussion}

The downward migration of stratospheric wind anomalies into the troposphere has been well observed in data and simulated in GCMs (e.g., Kodera et al. 1990; Baldwin and Dunkerton 1999). Despite the apparent robustness of this evidence, the principal mechanisms whereby stratospheric variability influences tropospheric circulation remain unclear. In view of this, the present study revisits the role of eddy and nonconservative processes in the downward migration of extratropical wind anomalies during SVW events by exploiting a new formulation of the theory of wave activity conservation and wave-mean flow interaction (Nakamura and Solomon 2010; Huang and Nakamura 2016, 2017).

There are several advantages to using the wave-mean flow interaction diagnostic based on finite-amplitude versions of wave activity to study stratosphere-troposphere dynamical coupling. First, it provides a precise link between wave activity and the driving of the mean flow that remains true at finite wave amplitude. This is because of the tendency of present wave activity to equal the (negative) EP flux divergence in the conservative limit even at finite amplitude. Second, the eddy effects on the mean flow can be more readily distinguished from nonconservative effects (such as eddy-induced mixing and diabatic heating). In addition, unlike traditional eddy-mean flow interaction theory that addresses how fast the mean flow is being modified by eddies (i.e., tendency), our diagnostic allowed us to quantify how much the mean flow is already modified by the eddies at any given time. Through application of this method, we can conclude the following:

1) The downward migration of extratropical wind anomalies is largely attributable to dynamical adjustments induced by fluctuating finite-amplitude wave forcing. The nonconservative effects, on the 

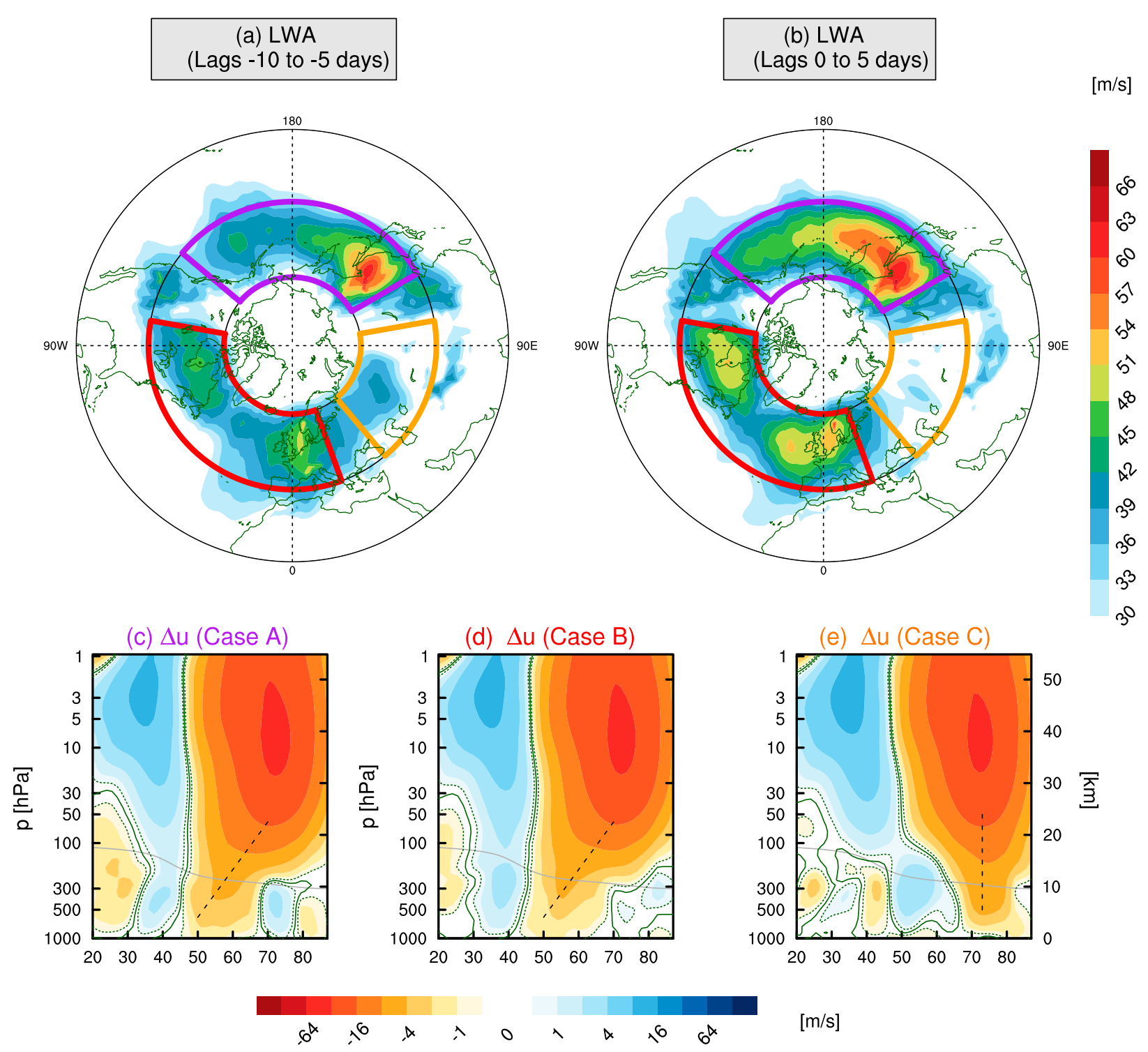

$[\mathrm{m} / \mathrm{s}]$

FIG. 9. (top) Column-averaged tropospheric local wave activity (250-1000 hPa) (a) before and (b) after the onset of the SVW events in the observations. The polygons indicate regions used for the inversions, including the North Pacific (case A: purple), the North Atlantic (case B: red), and the Eurasian (case C: orange) sectors. (bottom) Latitude-height cross section of the observed zonal wind anomalies induced by eddies $\Delta u$ in response to tropospheric local wave activity in (c) the North Pacific, (d) the North Atlantic, and (e) Eurasia during the onset of the events (lag 0 to +5 days). The gray solid lines in (c)-(e) indicate the approximate boundary between the troposphere and the stratosphere. The dashed lines indicate an approximate slope of the negative wind anomalies from about 50 to $850 \mathrm{hPa}$.

other hand, contribute to maintaining the downwardpropagating wind anomalies in the aftermath/recovery within the stratosphere but seem negligible in the tropospheric evolution.

2) Variations in stratospheric finite-amplitude wave forcing are too weak to account for the attendant changes and shapes in the tropospheric flow. Our results show that the indirect effect of tropospheric finite-amplitude wave activity through residual displacements is needed to amplify and prolong the tropospheric wind re- sponses. This suggests that the primary effect of tropospheric wave activity is to spin up the residual circulation, causing adjustment in the mean flow.

3) The dynamical adjustments induced by stratospheric $A^{*}$ are characterized by anomalous "quasi barotropic" wind patterns from the stratosphere to the upper troposphere at high latitudes, while the tropospheric $A^{*}$ is characterized by a northward-tilting pattern of the tropospheric wind anomaly between $45^{\circ}$ and $60^{\circ} \mathrm{N}$. 
4) The local tropospheric finite-amplitude wave activity over the North Pacific and North Atlantic sectors plays a significant role in shaping the structure of the high-latitude tropospheric wind response to SVW events. This indicates that the latitude-height structure of the tropospheric response is determined by the local structure of the tropospheric wave activity.

The first result demonstrates that the downward migration of the wind anomalies is, to first order, driven by the localized finite-amplitude wave-mean flow interaction, confirming the result of Plumb and Semeniuk (2003) based on idealized simulations. Here, we revisited the mechanism based on the observed wave activity $\left(A^{*}\right)$ conservation and wave-mean flow interaction. We show from Eq. (5), for a nearly barotropic $A^{*}$, the solution is $\Delta u \simeq-A^{*}$. Therefore, to the extent that $A^{*}$ descends with time (see Fig. 3), it is expected that $\Delta u$ will do the same (see Fig. 2). In reality, however, $\Delta u$ deviates significantly from $A^{*}$, reflecting the effects of meridional displacement due to residual circulation $\Delta u_{R}$. In particular, $\Delta u_{R}$ partially cancels out $A^{*}$ in the stratosphere, and it too descends with time (Fig. 3). This suggests that the nonlocal influence of wave activity on the zonal flow through the residual circulation (e.g., downward control) in the stratosphere is limited and mainly opposes the direct influence of $A^{*}$. In the troposphere, on the other hand, there is a greater degree of cancellation between $\Delta u_{R}$ and $-A^{*}$, and after a lag of 10 days, the roles of $\Delta u_{R}$ and $A^{*}$ are reversed (Fig. 3). Unlike in the stratosphere, it is the indirect influence of $A^{*}$ on the zonal flow through the residual displacement that mainly drives the deceleration of the negative zonal wind anomaly.

The results also demonstrate that the nonconservative processes may contribute to maintaining the stratospheric wind anomalies during the recovery period of SVW events. The mechanism can be related to significant increased eddy-induced mixing after the vortex breakdown (e.g., Allen and Nakamura 2001), which leads to stronger irreversible wave dissipation and thus weaker divergence of the EP flux. In a subsequent paper, we will show that increased irreversible dissipation, due to mixing and diabatic forcing during the recovery phase of the warming events, dampens the wave transience effects, resulting in delayed recovery of the polar vortex (Lubis et al. 2018, manuscript submitted to J. Atmos. $S c i$.). This suggests that nonconservative processes associated with mixing and diabatic heating are important in maintaining easterly wind anomalies in the stratosphere during the recovery period of SVW events.

Our results also help to clarify conclusions from previous studies based on idealized numerical experiments (Song and Robinson 2004; Charlton et al. 2005; Thompson et al. 2006; Kunz and Greatbatch 2013).
Some studies have argued that the tropospheric eddy feedbacks are needed to explain the tropospheric response to stratospheric variability (Song and Robinson 2004; Charlton et al. 2005; Kunz and Greatbatch 2013), while other studies claim that only stratospheric forcing (without tropospheric eddy feedbacks) can fully account for the attendant changes (amplitude) in the tropospheric flow (Thompson et al. 2006). Our results clarify that, while the dynamical adjustment induced by stratospheric eddy activity has a direct effect on the tropospheric wind, the nonlocal influence of the tropospheric eddy adjustments on the zonal-mean flow, through residual displacements, is required to amplify and maintain the tropospheric surface signals. In particular, we showed that, by imposing finite-amplitude stratospheric wave forcing in the stratosphere only, the tropospheric response is too weak to account for the total changes, suggesting that the observed response can only be realized with the additional contribution from tropospheric eddy momentum feedbacks (e.g., Kidston et al. 2015). Moreover, our results indicate that the upper-tropospheric eddy feedbacks maintain the surface signals for a few days after the onset (exhibiting a nearinstantaneous vertical coupling to the stratosphere), whereas the lower-tropospheric eddy feedbacks prolong that surface signal over several weeks. These results have not been clearly shown in previous studies.

The importance of local tropospheric wave activity in shaping the structure of the tropospheric response to SVW events is also emphasized in this study. We show that there are two dynamically active regions that are important in getting a correct representation of the tropospheric responses (both location and amplitude) to SVW events, which are located around the storm-track regions, over the North Pacific and North Atlantic basins. This result quantitatively supports the previous work (e.g., Hitchcock and Simpson 2014), arguing that latitude-longitude tropospheric dynamics are important in the tropospheric response to SVW events.

Overall, the results are found to be robust, in a qualitative sense, with the results from GCM simulations and do not depend on the details of the specific model configuration. Since our diagnostic does not require a reference state based on a zonal mean that changes with time (i.e., it does not interact with eddies), it provides an objective measure of finite-amplitude eddies in the observed stratosphere-troposphere connection. This diagnostic may, hence, be used as an objective tool for diagnosing and verifying the results of various numerical studies on stratosphere-troposphere coupling.

Acknowledgments. The authors thank two anonymous reviewers for their useful comments. This research 
is supported by National Science Foundation (NSF) Grant AGS-1563307. MJ acknowledges the support of the ARC Centre of Excellence for Climate System Science (CE110001028). We would also like to acknowledge high-performance computing support from Cheyenne (https://doi.org/10.5065/D6RX99HX) provided by NCAR's Computational and Information Systems Laboratory, sponsored by the NSF. We are also grateful for the support of the University of Chicago Research Computing Center (RCC) for the calculations and a model simulation carried out in this work. The data from all model integrations are available from the authors upon request.

\section{APPENDIX A}

\section{Adiabatic Surface Boundary Condition for the Inversion}

In this study, the adiabatic surface boundary condition was tested as an alternative lower boundary condition to find the most accurate approximation of $\Delta u$ from the inversion procedure. This boundary condition assumes that eddies are adiabatic near the surface, just as in the interior, and unconstrained by friction or heat exchange with the ground. The difference between the adiabatic and no-slip lower boundary conditions lies in the arrangement of angular momentum by an implied meridional displacement caused by surface friction.

Following Nakamura and Solomon (2010), the adiabatic surface boundary condition meets the following criteria:

$$
\frac{\partial}{\partial z} \frac{\Delta u \cos \phi_{e}}{f}=\frac{R\left(1-\sin ^{2} \phi_{e}\right)}{f^{2} a^{2} H} e^{-\kappa z_{0} / H} \frac{\partial^{2}\left(B \cos \phi_{e}\right)}{\partial\left(\sin \phi_{e}\right)^{2}},
$$

where

$$
B\left(\phi_{e}, t\right)=\frac{1}{2 \pi a \cos \phi_{e}}\left[\iint_{\theta_{s} \leq \Theta\left(\phi_{e}\right)} \theta_{s} d s-\iint_{0.5 \pi \geq \phi \geq \phi_{e}} \theta_{s} d s\right] .
$$

Here, $\kappa=R / c_{p}, c_{p}$ is the specific heat at constant pressure, $a$ is the radius of Earth, and $f$ is the Coriolis parameter. The surface wave activity $B$ is defined much the same as the interior wave activity $A^{*}$ [see Eq. (1) in the main text] but based on the surface potential temperature $\theta_{s}$.

This surface boundary condition is applied to the first level above the ground $\left(z_{0}=1 \mathrm{~km}\right)$. The results of such a test are shown in Fig. A1, which displays the net adiabatic adjustments by eddies $\Delta u$ and the nonconservative contributions $u_{\mathrm{REF}}$ to the $u$ anomalies for the composites of SVW events. It is shown that the assumed surface
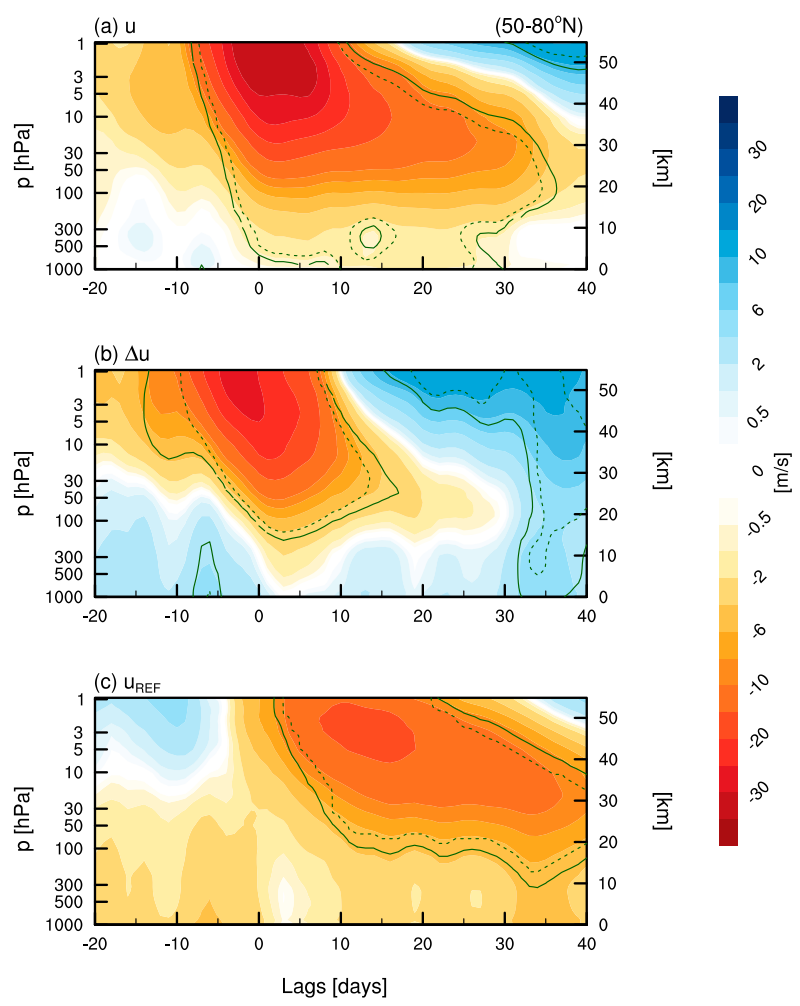

FIG. A1. As in Figs. 2a-c, respectively, but for the adiabatic surface boundary condition from ERA-Interim. The solid (dashed) contour lines indicate the $95 \%$ (99\%) significance level based on a 1000-trial Monte Carlo test.

boundary condition for the reference state has a profound effect on the structure of the adjustment (Fig. A1b; cf. Fig. $2 b$ in the main text). Under adiabatic surface boundary condition, the $\Delta u$ values are not significant in the troposphere and exhibit positive adjustments (acceleration) on the mean flow. After the onset of the events, the zonalmean flow induced by eddies in the troposphere is almost everywhere faster than the reference state (Fig. A1c), which is inconsistent with the observed tropospheric wind response (Fig. A1a). This suggests that the troposphere diabatic processes must decelerate flow substantially to explain the observed response of $\bar{u}$.

We should note that in reality the adiabatic surface boundary assumption is poor in the boundary layer, and this has a far-reaching impact on the stratosphere as well. The obtained reference state $u_{\mathrm{REF}}$ grossly overestimates the surface westerlies, which is incompatible with even a small amount of friction. In other words, it cannot be recovered as an inviscid limit of the no-slip boundary condition. That alone is sufficient to not choose the adiabatic boundary condition. A detailed comparison of the adiabatic and no-slip surface boundary conditions used for determining $\Delta u$ has been discussed thoroughly in Nakamura and Solomon (2010). 


\section{APPENDIX B}

\section{Statistical Significance Analysis}

We apply a nonparametric (Monte Carlo) approach to establish statistical significance at the two-sided $95 \%$ and 99\% levels (Reichler et al. 2012; Lubis et al. 2017). In this approach, we take 1000 random composite subsamples from the entire population, where each subsample element has the same size as the quantity to be tested. We repeat this procedure 1000 times to create a distribution that is the result of pure chance. The upper and lower $2.5(0.5)$ percentiles of this distribution can be thought of as statistically significant at $95 \%$ (99\%) confidence limits.

\section{REFERENCES}

Allen, D. R., and N. Nakamura, 2001: A seasonal climatology of effective diffusivity in the stratosphere. J. Geophys. Res., 106, 7917-7935, https://doi.org/10.1029/2000JD900717.

Ambaum, M. H. P., and B. J. Hoskins, 2002: The NAO tropospherestratosphere connection. J. Climate, 15, 1969-1978, https://doi.org/ 10.1175/1520-0442(2002)015<1969:TNTSC > 2.0.CO;2.

Andrews, D. G., J. R. Holton, and C. B. Leovoy, 1987: Middle Atmosphere Dynamics. International Geophysics Series, Vol. 40, Academic Press, 128 pp.

Baldwin, M. P., and T. J. Dunkerton, 1999: Propagation of the Arctic Oscillation from the stratosphere to the troposphere. J. Geophys. Res., 104, 30 937-30 946, https://doi.org/10.1029/ 1999JD900445.

—, and —, 2001: Stratospheric harbingers of anomalous weather regimes. Science, 294, 581-584, https://doi.org/ 10.1126/science. 1063315 .

—, D. B. Stephenson, D. W. J. Thompson, T. J. Dunkerton, A. J. Charlton, and A. O'Neill, 2003: Stratospheric memory and skill of extended-range weather forecasts. Science, 301, 636640, https://doi.org/10.1126/science.1087143.

Black, R. X., 2002: Stratospheric forcing of surface climate in the Arctic Oscillation. J. Climate, 15, 268-277, https://doi.org/ 10.1175/1520-0442(2002)015<0268:SFOSCI >2.0.CO;2.

Charlton, A. J., A. O'Neill, P. Berrisford, and W. A. Lahoz, 2005: Can the dynamical impact of the stratosphere on the troposphere be described by large-scale adjustment to the stratospheric PV distribution? Quart. J. Roy. Meteor. Soc., 131, 525-543, https://doi.org/10.1256/qj.03.222.

Dee, D. P., and Coauthors, 2011: The ERA-Interim reanalysis: Configuration and performance of the data assimilation system. Quart. J. Roy. Meteor. Soc., 137, 553-597, https://doi.org/ 10.1002/qj.828.

Gent, P. R., and Coauthors, 2011: The Community Climate System Model version 4. J. Climate, 24, 4973-4991, https://doi.org/ 10.1175/2011JCLI4083.1.

Hartley, D. E., J. T. Villarin, R. X. Black, and C. A. Davis, 1998: A new perspective on the dynamical link between the stratosphere and troposphere. Nature, 391, 471-474, https://doi.org/ $10.1038 / 35112$.

Haynes, P. H., M. E. McIntyre, T. G. Shepherd, C. J. Marks, and K. P. Shine, 1991: On the "downward control" of extratropical diabatic circulations by eddy-induced mean zonal forces. J. Atmos.
Sci., 48, 651-678, https://doi.org/10.1175/1520-0469(1991)048<0651: OTCOED $>2.0 . \mathrm{CO} ; 2$.

Hitchcock, P., and I. R. Simpson, 2014: The downward influence of stratospheric sudden warmings. J. Atmos. Sci., 71, 3856-3876, https://doi.org/10.1175/JAS-D-14-0012.1.

Holton, J. R., P. H. Haynes, M. E. McIntyre, A. R. Douglass, R. B. Rood, and L. Pfister, 1995: Stratosphere-troposphere exchange. Rev. Geophys., 33, 403-439, https://doi.org/10.1029/ 95RG02097.

Huang, C. S. Y., and N. Nakamura, 2016: Local finiteamplitude wave activity as a diagnostic of anomalous weather events. J. Atmos. Sci., 73, 211-229, https://doi.org/ 10.1175/JAS-D-15-0194.1.

_ and _ 2017: Local wave activity budgets of the wintertime Northern Hemisphere: Implication for the Pacific and Atlantic storm tracks. Geophys. Res. Lett., 44, 5673-5682, https://doi.org/ 10.1002/2017GL073760.

Jucker, M., S. Fueglistaler, and G. K. Vallis, 2013: Maintenance of the stratospheric structure in an idealized general circulation model. J. Atmos. Sci., 70, 3341-3358, https://doi.org/10.1175/ JAS-D-12-0305.1.

Kidston, J., A. A. Scaife, S. C. Hardiman, D. M. Mitchell, N. Butchart, M. P. Baldwin, and L. J. Gray, 2015: Stratospheric influence on tropospheric jet streams, storm tracks and surface weather. Nat. Geosci., 8, 433-440, https://doi.org/10.1038/ngeo2424.

Kodera, K., K. Yamazaki, M. Chiba, and K. Shibata, 1990: Downward propagation of upper stratospheric mean zonal wind perturbation to the troposphere. Geophys. Res. Lett., 17, 1263-1266, https://doi.org/10.1029/GL017i009p01263.

Kunz, T., and R. J. Greatbatch, 2013: On the northern annular mode surface signal associated with stratospheric variability. J. Atmos. Sci., 70, 2103-2118, https://doi.org/10.1175/JAS-D12-0158.1.

Limpasuvan, V., D. W. Thompson, and D. L. Hartmann, 2004: The life cycle of the Northern Hemisphere sudden stratospheric warmings. J. Climate, 17, 2584-2596, https://doi.org/10.1175/ 1520-0442(2004)017<2584:TLCOTN>2.0.CO;2.

Lubis, S. W., K. Matthes, N.-E. Omrani, N. Harnik, and S. Wahl, 2016: Influence of the quasi-biennial oscillation and sea surface temperature variability on downward wave coupling in the Northern Hemisphere. J. Atmos. Sci., 73, 1943-1965, https://doi.org/10.1175/JAS-D-15-0072.1.

_ V. Silverman, K. Matthes, N. Harnik, N.-E. Omrani, and S. Wahl, 2017: How does downward planetary wave coupling affect polar stratospheric ozone in the Arctic winter stratosphere? Atmos. Chem. Phys., 17, 2437-2458, https://doi.org/ 10.5194/acp-17-2437-2017.

_ Downward wave coupling between the stratosphere and troposphere under future anthropogenic climate change. J. Climate, https://doi.org/10.1175/JCLI-D-17-0382.1, in press.

Martineau, P., and S.-W. Son, 2015: Onset of circulation anomalies during stratospheric vortex weakening events: The role of planetary-scale waves. J. Climate, 28, 7347-7370, https://doi.org/ 10.1175/JCLI-D-14-00478.1.

Mitchell, D. M., L. J. Gray, J. Anstey, M. P. Baldwin, and A. J. Charlton-Perez, 2013: The influence of stratospheric vortex displacements and splits on surface climate. J. Climate, 26, 2668-2682, https://doi.org/10.1175/JCLI-D-12-00030.1.

Nakamura, N., 1996: Two-dimensional mixing, edge formation, and permeability diagnosed in an area coordinate. J. Atmos. Sci., 53, 1524-1537, https://doi.org/10.1175/1520-0469(1996)053<1524: TDMEFA $>2.0 . \mathrm{CO} ; 2$. 
- and A. Solomon, 2010: Finite-amplitude wave activity and mean flow adjustments in the atmospheric general circulation. Part I: Quasigeostrophic theory and analysis. J. Atmos. Sci., 67, 3967-3983, https://doi.org/10.1175/2010JAS3503.1.

_ and D. Zhu, 2010: Finite-amplitude wave activity and diffusive flux of potential vorticity in eddy-mean flow interaction. J. Atmos. Sci., 67, 2701-2716, https://doi.org/10.1175/2010JAS3432.1.

_ , and A. Solomon, 2011: Finite-amplitude wave activity and mean flow adjustments in the atmospheric general circulation. Part II: Analysis in the isentropic coordinate. J. Atmos. Sci., 68, 2783-2799, https://doi.org/10.1175/2011JAS3685.1.

Nielsen-Gammon, J. W., and R. J. Lefevre, 1996: Piecewise tendency diagnosis of dynamical processes governing the development of an upper-tropospheric mobile trough. J. Atmos. Sci., 53, 3120-3142, https://doi.org/10.1175/1520-0469(1996)053<3120: PTDODP $>2.0 . \mathrm{CO} ; 2$.

Perlwitz, J., and N. Harnik, 2003: Observational evidence of a stratospheric influence on the troposphere by planetary wave reflection. J. Climate, 16, 3011-3026, https:/doi.org/10.1175/ 1520-0442(2003)016<3011:OEOASI >2.0.CO;2.

Plumb, R. A., and K. Semeniuk, 2003: Downward migration of extratropical zonal wind anomalies. J. Geophys. Res., 108 , 4223, https://doi.org/10.1029/2002JD002773.

Reichler, T., J. Kim, E. Manzini, and J. Kroger, 2012: A stratospheric connection to Atlantic climate variability. Nat. Geosci., 5, 783-787, https://doi.org/10.1038/ngeo1586.
Scaife, A. A., and Coauthors, 2014: Skillful long-range prediction of European and North American winters. Geophys. Res. Lett., 41, 2514-2519, https://doi.org/10.1002/ 2014 GL059637.

Shaw, T. A., J. Perlwitz, and N. Harnik, 2010: Downward wave coupling between the stratosphere and troposphere: The importance of meridional wave guiding and comparison with zonal-mean coupling. J. Climate, 23, 6365-6381, https://doi.org/ 10.1175/2010JCLI3804.1.

Song, Y., and W. A. Robinson, 2004: Dynamical mechanisms for stratospheric influences on the troposphere. J. Atmos. Sci., 61, 1711-1725, https://doi.org/10.1175/1520-0469(2004) 061<1711:DMFSIO>2.0.CO;2.

Thompson, D. W. J., J. C. Furtado, and T. G. Shepherd, 2006: On the tropospheric response to anomalous stratospheric wave drag and radiative heating. J. Atmos. Sci., 63, 2616-2629, https://doi.org/10.1175/JAS3771.1.

Tripathi, O. P., and Coauthors, 2015: The predictability of the extratropical stratosphere on monthly time-scales and its impact on the skill of tropospheric forecasts. Quart. J. Roy. Meteor. Soc., 141, 987-1003, https://doi.org/10.1002/ qj.2432.

Wittman, M. A. H., L. M. Polvani, R. K. Scott, and A. J. Charlton, 2004: Stratospheric influence on baroclinic lifecycles and its connection to the Arctic Oscillation. Geophys. Res. Lett., 31, L16113, https://doi.org/10.1029/2004GL020503. 